\title{
ÁCIDO CÍTRICO E QUITOSANA NA CONSERVAÇÃO DE LICHIAS 'BENGAL' ${ }^{1}$
}

\author{
JOÃO EMMANUEL RIBEIRO GUIMARÃES², CRISTIANE MARIA ASCARI MORGADO², \\ VANESSA CURY GALATI ${ }^{4}$, KELLY MAGALHÃES MARQUES ${ }^{2}$, BEN-HUR MATTIUZ $^{3}$
}

RESUMO- Este trabalho teve como objetivo avaliar a aplicação de ácido cítrico em duas concentrações, associadas ou não à quitosana, na manutenção da qualidade de lichias 'Bengal'. Após a colheita e seleção, os frutos no estádio de maturação maduro foram imersos por 1 minuto nas seguintes soluções de ácido cítrico e de quitosana: [1] Testemunha - sem imersão; [2] ácido cítrico a $300 \mathrm{~g} \mathrm{~L}^{-1}$; [3] ácido cítrico a $300 \mathrm{~g} \mathrm{~L}^{-1}+$ $0,3 \%$ quitosana; [4] ácido cítrico a $300 \mathrm{~g} \mathrm{~L}^{-1}+0,6 \%$ quitosana; [5] ácido cítrico a $600 \mathrm{~g} \mathrm{~L}^{-1}$; [6] ácido cítrico a $600 \mathrm{~g} \mathrm{~L}^{-1}+0,3 \%$ quitosana; [7] ácido cítrico a $600 \mathrm{~g} \mathrm{~L}^{-1}+0,6 \%$ quitosana. Após a imersão, os frutos foram colocados em gôndolas para escorrer o excesso de solução. Em seguida, foram armazenados em câmara fria, previamente higienizada, a $5^{\circ} \mathrm{C}$, durante 20 dias. $\mathrm{O}$ experimento foi conduzido seguindo um delineamento inteiramente casualizado, num esquema fatorial composto por sete soluções de recobrimento e cinco datas de amostragem. A cada cinco dias, foi avaliada a perda de massa fresca dos frutos, a coloração, o teor de antocianinas e a atividade das enzimas polifenoloxidase e peroxidase da casca. A solução de ácido cítrico a $600 \mathrm{~g} \mathrm{~L}^{-1}$, associada ou não à quitosana, e a combinação de quitosana $0,3 \% \mathrm{com} 300 \mathrm{~g} \mathrm{~L}^{-1}$ de ácido cítrico foram as mais eficientes para a manutenção da cor avermelhada e a redução do escurecimento da casca de lichias 'Bengal' por 20 dias, a $5^{\circ} \mathrm{C}$.

Termos para indexação: Litchi chinensis Sonn., pós-colheita, qualidade, escurecimento.

\section{CITRIC ACID AND CHITOSAN IN CONSERVATION OF LITCHIS 'BENGAL'}

ABSTRACT - The aim of this study was to evaluate citric acid application with or without chitosan on the maintenance of the quality of litchi 'Bengal'. It was used litchi on mature maturation stage. After selection, fruits were immersed for one minute on the solution of citric acid and chitosan: [1]Control - without immersion; [2] citric acid 300g L $\mathrm{g}^{-1}$, [3] citric acid $300 \mathrm{~g} \mathrm{~L}^{-1}+$ chitosan 0,3\%, [4]citric acid $300 \mathrm{~g} \mathrm{~L}^{-1}+$ chitosan 0,6\%, [5] citric acid $600 \mathrm{~g} \mathrm{~L}^{-1}$, [6] citric acid $600 \mathrm{~g} \mathrm{~L}^{-1}+$ chitosan $0,3 \%$, [7] citric acid $600 \mathrm{~g} \mathrm{~L}^{-1}+$ chitosan $0,6 \%$. Chitosan of low molecular weight and deacetylation degree of 75,58\% (Sigma - Aldrich ${ }^{\circledR}$ ) were used. After immersion, fruits were put to drain the excess of solution. Then, they were stored in cool chamber at $5{ }^{\circ} \mathrm{C}$, previously sanitized, for 20 days. The experiment was conducted following a completely randomized design, with a factorial scheme composed by seven coating solutions and five sampling dates. Every five days were evaluated: loss of fresh mass of fruits; color; content of anthocyanins and the activity of peel enzymes polyphenoloxidase and peroxidase. It was verified that the solution of citric acid $600 \mathrm{~g} \mathrm{~L}^{-1}$, associated or not to chitosan, and the combination of chitosan $0,3 \%$ to $300 \mathrm{~g} \mathrm{~L}^{-1}$ of citric acid were the most efficient to maintain the quality of litchi 'Bengal' for 20 days at $5^{\circ} \mathrm{C}$.

Index terms: Litchi chinensis Sonn., postharvest, quality, darkening.

\footnotetext{
${ }^{1}$ (Trabalho 020-13). Recebido em: 03-01-2013. Aceito para publicação em: 16-09-2013.

${ }^{2}$ Alunos do Programa de Produção Vegetal da FCAV-UNESP, Câmpus de Jaboticabal. Via de Acesso Prof. Paulo Donato Castellane, s/n. CEP: 14.884-900. Jaboticabal-SP.E-mail: jerguimaraes@uol.com.br; cristianemorgado4@yahoo.com.br; vanessagalati@bol. com.br

${ }^{3}$ Professor do Departamento de Tecnologia da FCAV-UNESP, Câmpus de Jaboticabal. Via de Acesso Prof. Paulo Donato Castellane, s/n. CEP: 14.884-900. Jaboticabal-SP. E-mail: benhur@fcav.unesp.br
} 


\section{INTRODUÇÃO}

A lichia (Litchi chinensis Sonn.) pertence à família Sapindaceae e é originária da parte subtropical da China. Seu alto potencial comercial é devido ao seu sabor levemente acidificado, excelente aroma, elevado valor nutritivo e cor vermelha brilhante da casca (AGUILA et al., 2009).

Entretanto, após a colheita, os frutos apresentam rápido escurecimento e perda da coloração vermelha da casca, o que limita sua comercialização (AGUILA et al., 2009; HOJO et al., 2011). O escurecimento pós-colheita é resultado de um processo geralmente atribuído à degradação dos pigmentos vermelhos (antocianinas), associado com a oxidação de compostos fenólicos pelas enzimas polifenoloxidase (PPO) e/ou peroxidase (POD) (SOUZA et al., 2011).

Vários são os tratamentos sugeridos para manter a cor vermelha e reduzir o escurecimento do pericarpo de lichias, dentre os quais se destacam a imersão em soluções de ácidos (ascórbico, cítrico, dentre outros), quitosana, lecitina, ceras e compostos de enxofre e o acondicionamento em embalagens plásticas (HOJO, 2010). Os tratamentos com ácido são de grande interesse, pois mesmo ocasionando redução nos teores de antocianinas, estes tratamentos fazem com que a coloração da lichia (vermelha) se torne mais intensa ao longo do tempo (ZHANG et al., 2005).

A quitosana é uma forma desacetilada da quitina, solúvel em ácidos orgânicos, comestível e considerada segura para o uso na alimentação humana. Tem sido muito empregada em tratamentos de pós-colheita, pois apresenta propriedade "filmogênica", formando uma camada protetora sobre os frutos imergidos em suas soluções, auxiliando na redução da perda de água, modificando a atmosfera e reduzindo o amadurecimento dos frutos (SOUZA et al., 2011). Essas propriedades vêm sendo observadas em diversas frutas, como longans (JIANG; LI, 2001), lichias (HOJO et al., 2011), mangas (SOUZA et al., 2011), dentre outras. Em lichias, tratamentos com soluções de quitosana têm demonstrado retardo no escurecimento do pericarpo, limitando a perda de antocianina, flavonoides e compostos fenólicos, além de retardar a atividade da PPO e da POD (DUCAMPCOLLIN et al., 2008).

O objetivo do presente trabalho foi avaliar o efeito da aplicação de ácido cítrico, associada ou não à quitosana, na manutenção da qualidade de lichias 'Bengal'.

\section{MATERIAL E MÉTODOS}

Foram utilizadas lichias da variedade Bengal, adquiridas de pomar comercial localizado no município de Taquaritinga-SP. Os frutos foram colhidos pela manhã e selecionados quanto à uniformidade do estádio de maturação, considerando-se maduros aqueles com casca avermelhada, conforme padronização estabelecida para comercialização no mercado interno (SALOMÃO et al., 2006). As lichias utilizadas neste estudo tiveram os seguintes dados de coloração inicial: luminosidade entre 36 e 40, cromaticidade entre 45 e 51 e ângulo Hue entre 27 e 30.

Os frutos foram acondicionados em caixas plásticas revestidas com plástico bolha e cuidadosamente transportados por $25 \mathrm{~km}$, até o Laboratório de Tecnologia dos Produtos Agrícolas da FCAV/ UNESP, Jaboticabal-SP, onde foram novamente selecionados quanto ao tamanho, cor e ausência de injúrias, visando a maior uniformidade do lote. Após a seleção, os frutos foram higienizados, imersos por 5 minutos em solução de dicloroisocianurato de sódio di-hidratado (Sumaveg ${ }^{\circledR}$ ) na concentração de $0,66 \%$,

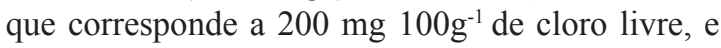
o excesso de água foi escorrido,em condição de ambiente $\left(22^{\circ} \mathrm{C} \pm 85 \%\right.$ UR). Em seguida, as lichias foram imersas, por 1 minuto, nas seguintes soluções de ácido cítrico e quitosana: [1] Testemunha - sem imersão; [2] ácido cítrico a $300 \mathrm{~g} \mathrm{~L}^{-1}$; [3] ácido cítrico $300 \mathrm{~g} \mathrm{~L}^{-1}+0,3 \%$ quitosana; [4] ácido cítrico $300 \mathrm{~g} \mathrm{~L}^{-1}+0,6 \%$ quitosana; [5] ácido cítrico a $600 \mathrm{~g}$ $\mathrm{L}^{-1} ;$ [6] ácido cítrico $600 \mathrm{~g} \mathrm{~L}^{-1}+0,3 \%$ quitosana; [7] ácido cítrico $600 \mathrm{~g} \mathrm{~L}^{-1}+0,6 \%$ quitosana. A quitosana utilizada no experimento possui baixo peso molecular e grau de desacetilização de 75,58\% (Sigma-Aldri$\left.\mathrm{ch}^{\circledR}, \operatorname{cod} .448869\right)$ ). Após a imersão, os frutos foram colocados para escorrer o excesso de solução em temperatura ambiente, a fim de formar o filme na superfície, em seguida, armazenados em câmara fria, previamente higienizada, a $5^{\circ} \mathrm{C}$, durante 20 dias.

$\mathrm{O}$ experimento foi conduzido seguindo um delineamento inteiramente casualizado, num esquema fatorial $7 \times 5$ : sete soluções de recobrimento e cinco datas de amostragem $(0 ; 5 ; 10 ; 15$ e 20 dias $)$. Foram utilizadas duas repetições, com 12 frutos cada.

A cada cinco dias, foi avaliada a perda de massa fresca dos frutos, utilizando-se de balança digital com capacidade para $2.000 \mathrm{~g}$ e precisão de $0,1 \mathrm{~g}$. Foi avaliada a coloração da casca no mesmo grupo de frutos até o final do experimento, utilizandose de colorímetro Minolta CR400, expressa em luminosidade (valores próximos de $0=$ preto; $100=$ branco) e cromaticidade (intensidade da cor), fornecidos pelo aparelho, pelos valores de "a" e "b", 
conforme a equação; o teor de antocianinas, expresso em mg $100 \mathrm{~g}^{-1}$ de casca, que foi determinado pelo método colorimétrico que utiliza como extrator a mistura de etanol e $\mathrm{HCl}$, deixando descansar por uma noite em geladeira, fazendo a leitura em espectrofotômetro em um comprimento de onda de $535 \mathrm{~nm}$; e a atividade das enzimas foi determinada conforme o proposto por Allain et al. (1974), sendo polifenoloxidase (PPO) com leitura espectrofotométrica em $420 \mathrm{~nm}$ e expressa em $\mu \mathrm{mol}$ de fenol consumido min $^{-1} \mathrm{~g}^{-1}$ e peroxidase (POD) em $505 \mathrm{~nm}$ expressa em nmol $\mathrm{H}_{2} \mathrm{O}_{2}$ consumido min ${ }^{1} \mathrm{~g}^{-1}$. Também foram avaliados os teores de sólidos solúveis, ácido ascórbico e acidez titulável, conforme metodologia AOAC (1997).

Os dados obtidos foram submetidos à análise de variância, pelo teste $\mathrm{F}$, e as médias, comparadas mediante o teste de Tukey, a 5\% de probabilidade. Havendo interação significativa entre os fatores, os resultados foram submetidos à regressão polinomial.

\section{RESULTADOS E DISCUSSÃO}

Não foram encontradas diferenças significativas entre os tratamentos para as análises de sólidos solúveis, ácido ascórbico e acidez titulável, apresentando valores médios de $19,11^{\circ} \mathrm{Brix}, 43,88$ mg de ácido ascórbico $100 \mathrm{~g}^{-1}$ e $0,31 \mathrm{~g}$ de ácido cítrico $100 \mathrm{~g}^{-1}$, respectivamente.

Quanto à luminosidade e à cromaticidade, houve interação significativa entre os tratamentos e os dias de armazenamento. Houve diminuição nos valores de luminosidade aos cinco dias, indicando escurecimento dos frutos, em todos os tratamentos (Figura 1). Isso corrobora Saengnil et al. (2006), que verificaram que as imersões de lichias em ácido ascórbico ou em ácido cítrico não foram eficazes na prevenção do escurecimento do pericarpo em condições de ambiente, pois os frutos tratados com este ácido mudaram a coloração em um dia para marrom, diminuindo assim sua luminosidade.

Esse escurecimento foi maior nos frutos do tratamento-Testemunha, onde se verificou diminuição progressiva da luminosidade até o final do armazenamento. Observou-se que, a partir do $5^{\circ}$ dia de armazenamento, as soluções de ácido cítrico a $600 \mathrm{~g} \mathrm{~L}^{-1}$, associadas ou não à quitosana e à combinação da solução de ácido cítrico a $300 \mathrm{~g} \mathrm{~L}^{-1}$ com $0,3 \%$ de quitosana, mantiveram os valores de luminosidade até o final do armazenamento.

Esse escurecimento, observado através da mudança da coloração vermelha para marrom, pode ocorrer até mesmo em frutos ligados à planta-mãe, em poucas horas ou até em três a quatro dias após a colheita (LIMA et al., 2010).

Comportamento similar foi observado para a variável cromaticidade, em que os valores para os frutos não tratados (Testemunha) diminuíram ao longo do período, diferenciando-se significativamente dos que receberam os demais tratamentos (Figura 2). Novamente, observou-se que as soluções de ácido cítrico a $600 \mathrm{~g} \mathrm{~L}^{-1}$, associado ou não à quitosana e a combinação de ácido cítrico a $300 \mathrm{~g} \mathrm{~L}^{-1}$ com $0,3 \%$ de quitosana, mantiveram a intensidade dos pigmentos, até o final do período de armazenamento. Desta maneira, pode-se inferir que estes tratamentos permitiram a manutenção da coloração vermelha característica das lichias por até 20 dias, quando armazenadas a $5^{\circ} \mathrm{C}$. Esses resultados foram semelhantes aos encontrados por Silva et al. (2011), em que frutos de lichias 'Bengal', armazenados a $5^{\circ} \mathrm{C}$, também mantiveram sua coloração em até 20 dias tratados com $\mathrm{HCl}$; já os frutos envoltos em filme de PVC mantiveram sua coloração por apenas quatro dias.

Trabalhando com lichias 'Bengal' tratadas com quitosana a $0,5 \%$ e armazenadas a $5^{\circ} \mathrm{C}$, Hojo et al. (2011) verificaram que a quitosana mostrou-se efetiva na manutenção da coloração vermelha e na prevenção do escurecimento, conservando a aparência dos frutos por até 20 dias, resultados coerentes com os encontrados neste trabalho.

Ducamp-Collin et al. (2008), ao armazenarem lichias das cultivares Kwai e Guiwei, a $4^{\circ} \mathrm{C}$ e $90 \%$ $\mathrm{UR}$, tratadas com ácido cítrico a $600 \mathrm{~g} \mathrm{~L} \mathrm{~L}^{-1}$ mais $0,75 \%$ de quitosana, verificaram aumento na vida de prateleira destes frutos em três semanas, quando comparados com os frutos não tratados, que passaram rapidamente da coloração vermelha para a marrom. Os autores ressaltam que o fator limitante para este tipo de tratamento foi a viscosidade da solução de ácido cítrico, que, nesta concentração e associada à quitosana, dificultou e aumentou o tempo de secagem após a imersão, o que também foi observado neste trabalho.

A mudança da coloração do pericarpo da lichia, de vermelho intenso para marrom, acontece devido a um rápido decréscimo da luminosidade (L*), cuja consequência imediata é a perda de seu valor comercial ou uma forte redução do preço de comercialização destes frutos no varejo (SILVA et al., 2011).

Verificou-se que a atividade da polifenoloxidase (PPO) aumentou até o $10^{\circ}$ dia de armazenamento, com posterior redução (Tabela 1). Este incremento também foi relatado por Ledsham (1994) em lichias 'Brewster' tratadas com ácido ascórbico a 0 e $10 \%$, cujo pico de atividade foi no primeiro dia 
de armazenamento e redução após o quarto dia. Com relação aos tratamentos utilizados, notou-se que, apesar de não ocorrerem diferenças significativas, os frutos tratados tiveram valores superiores aos da Testemunha (Tabela 1).

Apesar de ocorrerem diferenças significativas somente no primeiro dia de armazenamento, notou-se incremento da ação da peroxidase (POD) ao longo do armazenamento (Tabela 1). Os incrementos nas atividades das enzimas da PPO e POD não foram suficientes para promover o escurecimento dos frutos, fato que pode ser explicado pelo efeito da quitosana na captação do oxigênio e proteção, formando uma barreira que impede a difusão do oxigênio para o interior do produto, reduzindo as quinonas geradas e inibindo a ação destas enzimas (REUCK et al., 2011), contribuindo para a manutenção da coloração vermelha da casca.

Observou-se que a enzima POD teve comportamento similar à atividade da PPO, não ocorrendo diferenças significativas entre os tratamentos.

Hojo (2010) relaciona o escurecimento do pericarpo com a degradação das antocianinas através da atividade da PPO e da POD, o que não foi observado neste trabalho.

Segundo Ducamp-Collin et al. (2008), a atividade da PPO e POD na casca de lichias diferem quanto à cultivar. Estes autores relatam que lichias da cultivar cheeWai,tratadas com ácido cítrico e quitosana, tiveram a atividade da PPO aumentada em mais de oito vezes e a da POD, em 20 vezes, enquanto na variedade Kwai houve incremento da PPO em 15\% e não foi constatada atividade enzimática da POD.

Observou-se redução significativa no teor de antocianinas das lichias ao longo do período de armazenamento, sendo que as maiores degradações foram encontradas nos frutos não tratados (Figura 3). Verificou-se, também, que a maior perda de antocianina nas lichias da Testemunha foi a partir do $5^{\circ}$ dia de armazenamento, coincidindo com o período em que as enzimas PPO e POD mostraram as maiores atividades. Zhang et al. (2005) também observaram que os teores de antocianinas diminuem com o aumento do escurecimento da casca e com o tempo de armazenamento. Embora os pericarpos diminuam seus teores de antocianina, observou-se que, após os tratamentos com ácidos, a coloração vermelha da lichia manteve-se ao longo do período de armazenamento, pois, como a maioria dos pigmentos naturais, as antocianinas apresentam instabilidade, sendo normalmente mais estáveis sob condições ácidas (JACKMAN; SMITH, 1992). Sendo assim, tratamentos ácidos em frutas de coloração vermelha são de grande interesse para a estabilidade da coloração.

Houve perda de massa fresca ao longo do armazenamento, sendo que as maiores foram verificadas nos frutos não tratados (Figura 4). Isto provavelmente está associado às reações metabólicas da respiração e com a transpiração do produto, que reduzem a quantidade de água presente no tecido vegetal, uma vez que os frutos não tratados não apresentavam barreiras semipermeáveis formadas pela aplicação de quitosana.

É possível verificar que todos os tratamentos, com exceção da Testemunha, foram eficientes em diminuir a perda de massa fresca (Figura 4), destacando-se a solução de ácido cítrico a $600 \mathrm{~g} \mathrm{~L}^{-1}$ que, independentemente da associação com quitosana, foi a que levou às menores perdas, juntamente com a combinação de ácido cítrico a $300 \mathrm{~g} \mathrm{~L}^{-1} \operatorname{com} 0,3 \%$ de quitosana (Figura 4). Cabe destacar que, aos 20 dias de armazenamento, o valor médio de perda de massa fresca para todos os tratamentos foi de $10,85 \%$. Esse valor foi inferior aos encontrados por Silva et al. (2010), em frutos de lichias 'Bengal' tratadas com diferentes doses de ácido ascórbico e armazenadas a $5^{\circ} \mathrm{C}$, por 12 dias $(16,8 \%)$.

Hojo et al. (2011) também encontraram valores superiores $(18,51 \%)$ em lichias tratadas com quitosana a $0,5 \%$ e armazenadas durante 24 dias, a $5^{\circ} \mathrm{C}$. Segundo Silva et al. (2011), perdas de massa fresca acima de $6,6 \%$ resultam em escurecimento do pericarpo de lichias. Isto reafirma que os tratamentos com solução de ácido cítrico a $600 \mathrm{~g} \mathrm{~L}^{-1}$, com ou sem quitosana, e a combinação ácido cítrico a $300 \mathrm{~g} \mathrm{~L}^{-1}$ com $0,3 \%$ de quitosana foram melhores, pois apresentaram perdas menores que os valores mencionados acima.

Neste experimento, foi possível observar os efeitos benéficos principalmente da aplicação de ácido cítrico na manutenção da cor vermelha e na redução do escurecimento em pericarpo de lichias, constituindo-se em um tratamento pós-colheita eficaz e de baixo custo, além de não oferecer riscos ao consumidor e ao meio ambiente. 


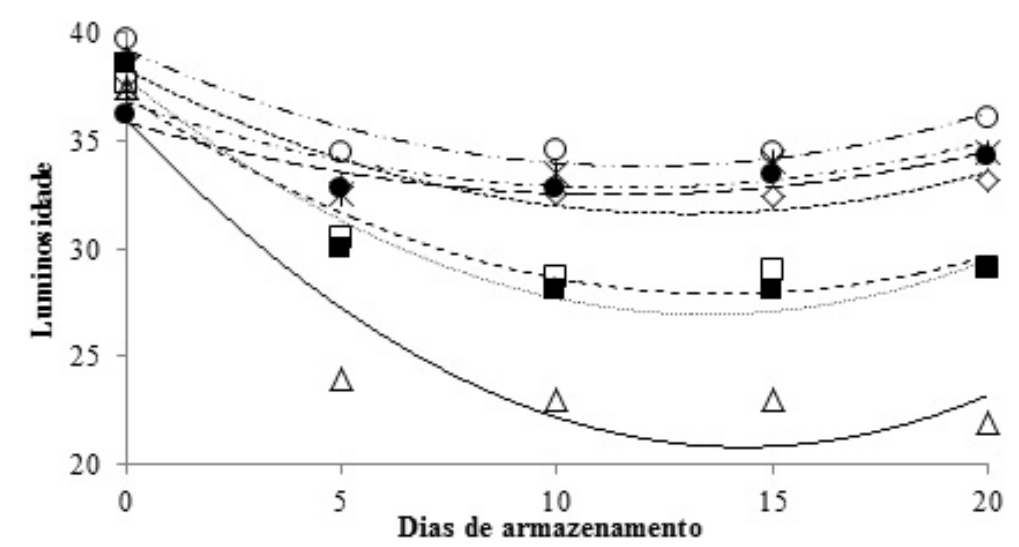

\footnotetext{
$y(\Delta$ Testemunha $)=0,074 x^{2}-2,125 x+36,01 R^{x}=0,885 \quad y(+A 600)=0,03 x^{2}-0,69 x+36,8 R^{x}=0,697$

$y(\cdot A .300)=0,06 x^{2}-1,62 x+37,9 R^{2}=0,959 \quad y(\bullet A, 600+Q 0,3)=0,026 x^{2}-0,595 x+35,87 R^{z}=0,865$

$y(0 A .300+Q 0,3)=0,039 x^{2}-1,023 x+38,29 R^{2}=0,904 \quad y(\circ A, 600+Q 0,6)=0,037 x^{2}-0,904 x+39,22 R^{x}=0,897$

$y(a A, 300+Q 0,6)=0,047 x^{2}-1,321 x+37,08 R^{2}=0,946$
}

FIGURA 1- Luminosidade da casca de lichias 'Bengal' tratadas com ácido cítrico, associado ou não à

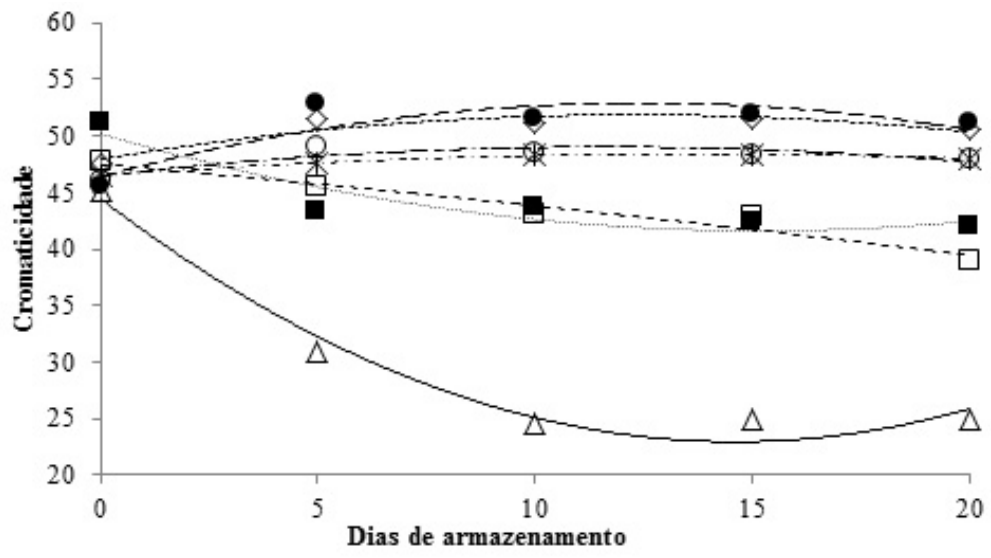

$\mathrm{y}(\triangle$ Testemunha $)=0,1007 \mathrm{x}^{2}-2,9401 \mathrm{x}+44,404 \mathrm{R}^{2}=0,9773$ $\mathrm{y}(\cdot \mathrm{A} 300)=0,0371 \mathrm{x}^{2}-1,1273 \mathrm{x}+50,158 \mathrm{R}^{2}=0,8629$ $y(\nabla A 300+Q 0,3)=-0,0259 x^{2}+0,6365 x+47,942 R^{2}=0,8471$ $y(\square A 300+Q 0,6)=-0,0035 x^{2}-0,3331 x+47,482 R^{2}=0,938$

$y(* A 600)=-0,0102 x^{2}+0,279 x+46,469 R^{2}=0,9985$

$\mathrm{y}(\bullet \mathrm{A} 600+\mathrm{Q} 0,3)=-0,0418 \mathrm{x}^{2}+1,0426 \mathrm{x}+46,401 \mathrm{R}^{2}=0,7623$

$y(\circ A, 600+Q 0,6)=-0,0189 x^{2}+0,4399 x+46,461 R^{2}=0,7208$

quitosana, e armazenadas a $5^{\circ} \mathrm{C}$.

FIGURA 2- Cromaticidade da casca de lichias 'Bengal' tratadas com ácido cítrico, associado ou não à 


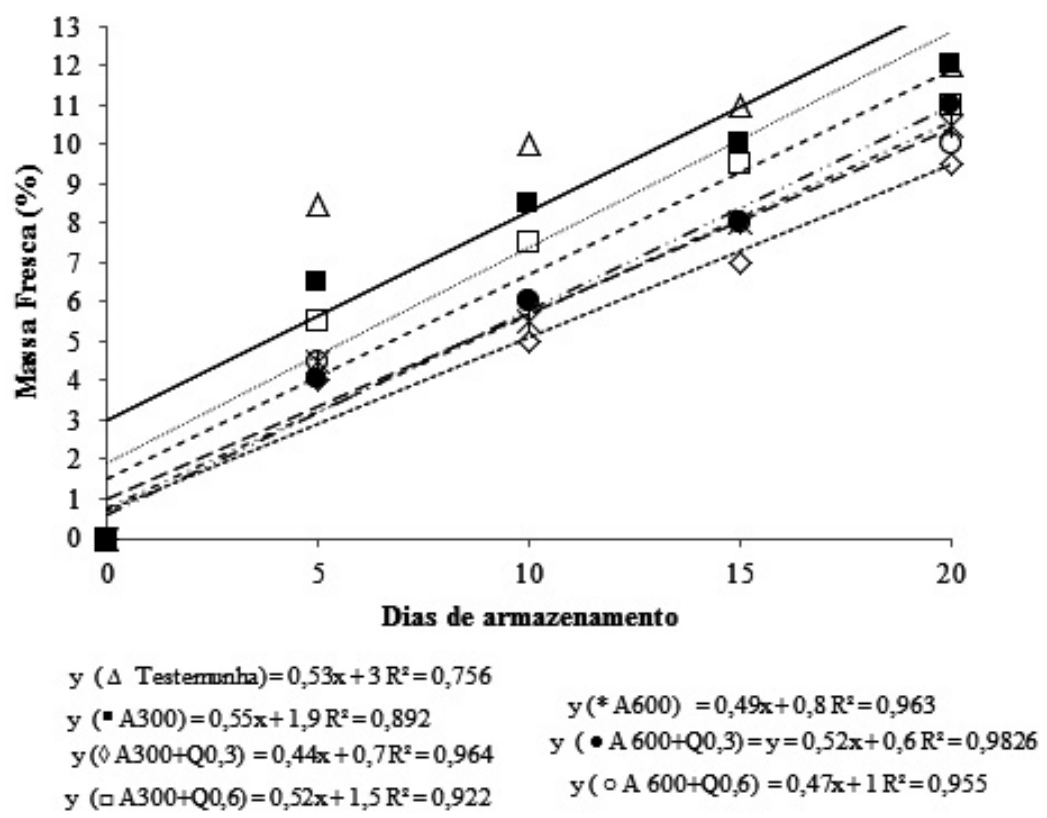

FIGURA 3 - Teores de antocianinas em mg $100 \mathrm{~g}^{-1}$, na casca de lichias 'Bengal', tratadas com ácido cítrico, associado ou não à quitosana, e armazenadas a $5^{\circ} \mathrm{C}$.

\section{CONCLUSÃO}

A solução de ácido cítrico a $600 \mathrm{~g} \mathrm{~L}^{-1}$, associada ou não à quitosana, e a combinação de ácido cítrico a $300 \mathrm{~g} \mathrm{~L}^{-1}$ mais $0,3 \%$ de quitosana foram os tratamentos mais eficientes para a manutenção da cor vermelha e a redução do escurecimento de lichias 'Bengal' por 20 dias, a $5^{\circ} \mathrm{C}$.

\section{REFERÊNCIAS}

AGUILA, J. S.; HOFMAN, P.; CAMPBELL, T.; MARQUES, J. R.; DEL AGUILA, L. S. H.; KLUGE, R. A. Pré-resfriamento em água de lichia 'B3' mantida em armazenamento refrigerado. Ciência Rural, Santa Maria, v. 39, n. 8, p. 2373-2379, 2009.

ALLAIN, C. C.; POON, L. S.; CHAN, C. S. G.; RICHMOND, W.; FU, P.C. Enzymatic determination of total serum colesterol. Clinical Chemistry, Baltimore, v. 120, p. 470-475, 1974.

AOAC. Official methods of analysis of the Association of Official Analytical Chemists International. $16^{\text {th }}$ ed. Washington: Ed. Patrícia Cunniff, 1997. v.2, 850p.
DUCAMP-COLLIN, M.; RAMARSON, H.; LEBRUN, M.; SELF, G.; REYNES, M. Effect of citric and chitosan on maintaining red coloration of litchi fruit pericarp. Postharvest Biology and Technology, Amsterdam, v. 49, p. 241-246, 2008.

HOJO, E. T. D. Aplicação de métodos combinados na conservação da qualidade de lichias 'Bengal'. 2010. 120f. (Doutorado em Agronomia) - Faculdade de Ciências Agrárias e Veterinárias, Universidade Estadual Paulista, Jaboticabal, 2010.

HOJO, E. T. D.; DURIGAN, J. F.; HOJO, R. H. Uso de embalagens plásticas e cobertura de quitosana na conservação pós-colheita de lichias. Revista Brasileira de Fruticultura, Jaboticabal, v. 33, n.1 p.377-383, 2011. Volume Especial.

JACKMAN, R. L.; SMITH, J. L. Anthocyanins and betalains. In: HENDRY, G. A. F.; HOUGHTON, J. D. Natural food colornts. New York: AVI, 1992.

JIANG, Y.; LI, Y. Effects of chitosan coating on phostharvest life and quality of longan fruit. Food Chemistry, London, v. 73, p. 139-143, 2001. 


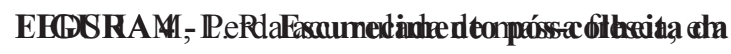
casca e qualipdardentensor;ide dehfunt bengalichria

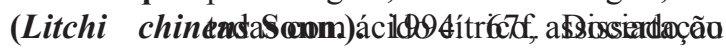

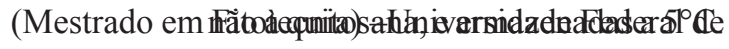
Viçosa, Viçosa, 1994.

LIMA, R. A. Z.; ABREU, C. M. P.; ASMAR, S. A.; CORRÊA, A. D.; SANTOS, C. D. Embalagens e recobrimento em lichias (Litchi chinensis Sonn.) armazenadas sob condições não controladas. Ciência e Agrotecnologia, Lavras, v. 34, n. 4, p. 914-921, 2010.

REUCK, K.; SIVAKUMAR, D.; KORSTEN, L. Integrated application of 1-methylcyclopropene and modified atmosphere packaging to improve quality retention of litchi cultivars during storage. Postharvest Biology and Technology, Amsterdam, v.52, n.1, p.71-77, 2011.

SAENGNIL, K.; LUEANGPRASERT, K.; UTHAIBUTRA, J. Control of enzymatic browning of harvested 'Hong Huay' litchi fruit with hot water and oxalic acid dips. Science Asia, Shangai, v. 32, p. 345-350, 2006.

SALOMÃO, L. C. C.; SIQUEIRA, D. L.; PEREIRA, M. E. C. Desenvolvimento do fruto da lichieira (Litchi chinensis Sonn.) 'Bengal'. Revista Brasileira de Fruticultura, Jaboticabal, v. 28, n.1, p. 11-13, 2006.
SILVA, D. F. P.; CABRINI, E. C.; ALVES, R. R.; SALOMÃO, L. C. C. Uso do ácido ascórbico no controle do escurecimento do pericarpo de lichia. Revista Brasileira de Fruticultura, Jaboticabal, v. 32, n. 2, p. 618-627, 2010.

SILVA, D. F. P.; SALOMÃO, L. C. C.; CABRINI, E. C.; ALVES, R. R.; STRUIVING, T. B. Prevenção do escurecimento do pericarpo de lichia através do uso de ácidos e filmes. Revista Brasileira de Fruticultura, Jaboticabal, v. 33, n.1 p. 519-527, 2011.

SOUZA, M. L.; MORGADO, C. M.A.; MARQUES, K. M.; MATTIUZ, C. F. M; MATTIUZ, B. H. Póscolheita de mangas 'Tommy Atkins' recobertas com quitosana, Revista Brasileira de Fruticultura, Jaboticabal, v. 33, n.1 p. 337-343, 2011. Volume Especial.

ZAUBERMAN, G.; RONEN, R.; AKERMAN, M.; WEKSLER, A.; ROT, I.; Fuchs, Y. Post-harvest retention of the red colour of litchi pericarp. Scientia Horticulturae, Amsterdam, v.47, p. 89-97, 1991.

ZHANG, Z.; PANG, X.; XUEWU, D.; JI, Z.; JIANG, $Y$. Role of peroxidase in anthocyanin degradation in litchi fruit pericarp. Food Chemistry, Kidlington, v. 90, p. 47-52, 2005. 